\section{(2) \\ BRAZILIAN JOURNAL \\ OF MEDICAL AND BIOLOGICAL RESEARCH}

www.bjournal.com.br
ISSN 1414-431X

Volume 45 (11) 995-1101 November 2012

BIOMEDICAL SCIENCES

AND

CLINICAL INVESTIGATION

Braz J Med Biol Res, November 2012, Volume 45(11) 1086-1094

doi: 10.1590/S0100-879X2012007500127

\title{
Association of postalimentary lipemia with atherosclerotic manifestations
}

J. Tentor, R.T. Nakamura, M. Gidlund, S. Barros-Mazon, L.M. Harada, V.S. Zago, J.F. Oba and E.C. de Faria

The Brazilian Journal of Medical and Biological Research is partially financed by

\section{且CNPq}

Ministério

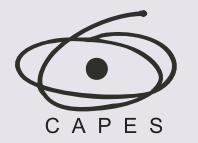

Ministério da Educação

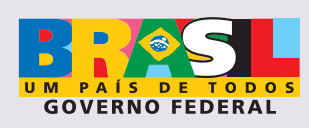

DTFAPESP

Institutional Sponsors

$\oplus$ SHIMADZU UNICAMP

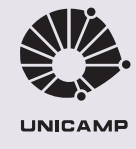

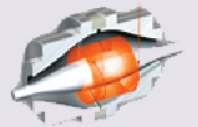

1DI Associaçăo Explore High - Performance MS Orbitrap Technology In Proteomics \& Metabolomics analitica $\underset{\text { analiticaweb.com.br }}{\text { Thermo }}$
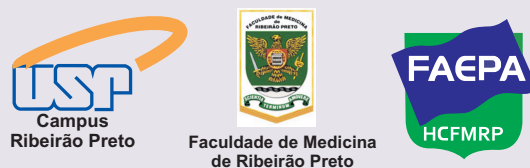


\title{
Association of postalimentary lipemia with atherosclerotic manifestations
}

\author{
J. Tentor ${ }^{1}$, R.T. Nakamura ${ }^{3,4}$, M. Gidlund ${ }^{5}$, S. Barros-Mazon ${ }^{1}$, L.M. Harada ${ }^{2}$, \\ V.S. Zago ${ }^{1}$, J.F. Oba ${ }^{1}$ and E.C. de Faria ${ }^{1}$ \\ ${ }^{1}$ Departamento de Patologia Clínica, Faculdade de Ciências Médicas, \\ Universidade Estadual de Campinas, Campinas, SP, Brasil \\ ${ }^{2}$ Laboratório de Lípides, Faculdade de Medicina, Universidade de São Paulo, São Paulo, SP, Brasil \\ ${ }^{3}$ Laboratório de Diagnóstico por Imagem, Campinas, SP, Brasil \\ ${ }^{4}$ Departamento de Radiologia, Universidade Estadual de Campinas, Campinas, SP, Brasil \\ ${ }^{5}$ Laboratório de Imunofisiopatologia, Instituto de Ciências Biológicas, \\ Universidade de São Paulo, São Paulo, SP, Brasil
}

\begin{abstract}
We identified different lipemic and metabolic responses after the ingestion of a standardized meal by healthy adults and related them to atherosclerotic markers. Samples from 60 normolipidemic adults were collected before and after a liquid meal (40 $\mathrm{g}$ fat $/ \mathrm{m}^{2}$ body surface) at $0,2,4,6$, and $8 \mathrm{~h}$ for measurements of lipids, free fatty acids (FFA), insulin, cholesteryl ester transfer protein (CETP), autoantibodies to epitopes of oxidized LDL (oxLDL Ab), lipolytic activities, and apolipoprotein E polymorphism. Mean carotid intima-media thickness (cIMT) was determined by Doppler ultrasound. The volunteers were classified into early $(\mathrm{N}=39)$ and late $(\mathrm{N}=31)$ triacylglycerol $(T A G)$ responders to the test meal. Late responders showed lower HDL cholesterol concentration at fasting and in the TAG peak, lower insulin and higher FFA concentrations compared to early responders. Multivariate regression analyses showed that mean cIMT was associated with gender (male) and age in early responders and by cholesterol levels at the 6th hour in late responders. oxLDL Ab were explained by lipoprotein lipase and negatively by hepatic lipase and oxLDL Ab (fasting period) by CETP (negative) and FFA (positive). This study is the first to identify a postalimentary insulin resistance state, combined with a reduced CETP response exclusively among late responders, and the identification of the regulators of postalimentary atherogenicity. Further research is required to determine the metabolic mechanisms described in the different postalimentary phenotypes observed in this study, as well as in different pathological states, as currently investigated in our laboratory.
\end{abstract}

Key words: Postalimentary lipemia; Autoantibodies to epitopes of oxidized LDL; Carotid intima-media thickness; Insulin; Free fatty acids

\section{Introduction}

Hypertriglyceridemia has been implicated in the risk of atherosclerosis-related cardiovascular events (1). Evidence from meta-analysis of a number of clinical studies in a large number of patients has established the increased concentration of plasma triacylglycerol (TAG) as an independent risk factor for coronary heart disease (CHD) (2). It is known that elevated fasting serum TAG concentration is a marker for atherogenic remnant lipoproteins and apolipoprotein Bcontaining lipoproteins or for other lipids and non-lipid risk factors (3). Epidemiologic data indicate that non-fasting TAG levels also are significantly associated with cardiovascular event rates $(4,5)$. The finding of TAG-rich lipoproteins in hu- man atheroma has provided substantial pathophysiologic indication of their direct role in atherogenesis (6). TAGrich lipoproteins are able to penetrate the arterial wall and reach the subendothelial space causing endothelial lipid deposits, attraction of monocytes, production of inflammatory markers, and oxidative stress. The role of TAG in the atherosclerosis-mediated inflammation depends not only on their direct effect on the endothelium, but also on attenuating the protective effects of high-density lipoprotein (HDL) against vascular inflammation (7).

Postalimentary lipemia was first described as an atherogenic phenomenon by Zilversmit in 1979 (8). Later,

Correspondence: E.C. de Faria, Departamento de Patologia Clínica, FCM, UNICAMP, Rua Tessália Vieira de Camargo, 126, 13084-971 Campinas, SP, Brasil. Fax: +55-19-3521-9434. E-mail: cottadefaria@gmail.com and/or cotta@fcm.unicamp.br

Received November 15, 2011. Accepted July 20, 2012. Available online August 10, 2012. Published October 5, 2012. 
it received more attention after the discovery that TAG-rich lipoproteins are atherogenic (9) and that patients with established CHD also have postprandial abnormalities in TAG metabolism, such as elevated concentrations and prolonged postprandial response after a fatty meal (10).

In Western societies, people spend most of their days in the postalimentary state because they have sequential eating habits. The magnitude of lipemia and of TAG peaks, the number of peaks, the time from baseline values to initial response, the lag time to peak, and the time to return to baseline values vary among individuals and are important components of the atherogenicity of the postalimentary state $(11,12)$. The studies found in the literature have not explored the metabolic background involved in the lipemic responses.

Clinical studies have shown an independent relationship between lipemia and early atherosclerosis as shown by the carotid intima-media thickness (cIMT) correlation with TAG at 1 to $4 \mathrm{~h} \mathrm{(11)} \mathrm{or} \mathrm{at} 6$ and $7 \mathrm{~h}(12)$. Moreover, patients with established coronary atherosclerosis present elevated TAG concentrations in the postalimentary state (12). Thus, the ultrasonic cIMT procedure can be used as an atherosclerosis marker to assess sub-clinical stages of the disease and predict coronary atherosclerosis (13).

Autoantibodies to epitopes of oxidized low-density lipoprotein (oxLDL Ab) are reported to play important roles in atherosclerosis, which monitor the degree of oxidative changes in LDL (14). These antibodies have been found in human plasma, as well as in human atherosclerotic lesions and in laboratory animals.

The objective of the present study was to measure the lipemic responses to a fat meal, metabolic components associated with it such as free fatty acids (FFA), insulin and cholesteryl ester transfer protein (CETP), and to determine their relationships with atherosclerosis biomarkers such as titers of oxLDL Ab and mean cIMT. To address this topic, we examined the postalimentary TAG responses of 60 normolipidemic asymptomatic adults.

\section{Material and Methods}

\section{Experimental protocol}

Sixty volunteers, 29 women (48\%) and 31 men (52\%), aged 18 to 45 years were selected for this study using the following criteria: all clinically asymptomatic, without any established CHD or cardiovascular risk factors such as the presence of dyslipidemia, hypertension, smoking, family history of myocardial infarction or sudden death, and diabetes mellitus, according to the recommendations of the National Cholesterol Education Program (15).

The protocol was approved by the Ethics Committee of the Faculdade de Ciências Médicas, Universidade Estadual de Campinas, São Paulo State. All participants gave written informed consent.

After an overnight fast (hereafter called baseline or fasting period, FP), blood was drawn into tubes containing $1 \mathrm{mg} / \mathrm{mL}$ EDTA. The volunteers received a lactose-free milkshake liquid meal (57\% carbohydrate, $13 \%$ protein, $25 \%$ fat, $9.8 \mathrm{~g} \%$ saturated fat, $4 \mathrm{~g} \%$ linoleic acid, $0.5 \mathrm{~g} \%$ linolenic acid, and $10 \mathrm{mg}$ cholesterol), providing $40 \mathrm{~g}$ fat/ $\mathrm{m}^{2}$ body surface (estimated from height and weight) over a period of up to $10 \mathrm{~min}$ (approximately $350 \mathrm{~mL}$ ). Additional blood samples were collected after 2, 4, 6, and $8 \mathrm{~h}$, hereafter called postalimentary period. Insulin, CETP and oxLDL Ab were measured during fasting and in the TAG peak period (highest TAG concentration after the meal). Lipoprotein lipase (LPL) and hepatic lipase (HL) activities were analyzed only during the fasting period. The other parameters were measured at all times.

After 2 to 3 days, the participants returned for a second visit to have fasting plasma collected for the quantification of lipases, 15 min after an intravenous heparin injection (100 U/kg body weight); up to 6 days after the first visit they came for a third visit for ultrasonographic examination of the carotid.

\section{Analytical methods}

Cholesterol, TAG and glucose were determined using enzymatic colorimetric methods and plasma HDL and LDL cholesterol were determined by homogeneous enzymatic colorimetric assays, all provided by Roche Diagnostics (Switzerland).

FFA were measured by an enzymatic method (Waco, Japan) and insulin by an immunometric assay (Immulite/ DPC/Medlab, USA).

LPL and HL activities were measured in post-heparin plasma samples on the basis of fatty acid release, using a radiolabeled triolein emulsion as the substrate and $\mathrm{NaCl}$ $(1 \mathrm{M})$ as the LPL inhibitor (16).

CETP activity was determined by an exogenous assay that measures the transfer of radiolabeled cholesteryl ester between a normal donor pool of ${ }^{14} \mathrm{CE}-\mathrm{HDL}$ and an unlabeled acceptor mixture of very LDL (VLDL) plus LDL over $4 \mathrm{~h}$, using plasma as the CETP source (17), and the results are reported as percent cholesteryl ester transferred. The inter-assay coefficients of variation were 11, 15, and $12 \%$ for CETP, LPL, and HL, respectively.

The chemical modification of LDL generates a variety of modified lipids and proteins that represent highly immunogenic neo-determinants. The IgG antibodies against holo-oxidized LDL were evaluated by ELISA after samples were incubated with copper-oxidized LDL (18). The results are presented as percent absorbance readings relative to the group ranked values.

Genomic DNA was obtained from peripheral blood cells by conventional methods (19). Restriction analysis of the PCR-amplified apolipoprotein $E$ genotypes (ApoE gene) polymorphism was performed as previously reported (20).

High-resolution B-mode carotid ultrasonography was 
performed by a single trained sonographer, who was blind to the subject's identity, using a 6-9-MHz linear array ultrasound imaging system (ATL HDI 1500 and 3500 Ultrasound System, Advanced Technology Laboratories Ultrasound, USA). The far wall of the distal $1 \mathrm{~cm}$ of the left and right common carotid arteries was scanned according to a standardized method (21). The mean common carotid intima-media thickness was calculated as the average of five measurements on each side (right and left) and expressed in $\mathrm{mm}$. The individual results were reported as the average of the left and right cIMT in $\mathrm{mm}$.

\section{Statistical analyses}

The statistical analyses were performed using visual inspection of the curves and the SPSS statistical package 6.0 (SPSS Inc., USA). The areas under the curves (AUC) and areas under the incremental curves (AUIC) were measured by the trapezoidal rule. Average differences were determined by one-way ANOVA followed by the post hoc Duncan test or the chi-square test, and the Wilcoxon test was used for comparison of fasting and postalimentary periods. Univariate and multivariate regression analyses were performed with the SAS for Windows software (Statistical Analysis System), 9.1.3, to understand which parameters were associated with mean cIMT, FP oxLDL Ab and TAG peak oxLDL $A b$ in the early and late groups, using stepwise criteria for variable selection and transforming the numerical variables into ranks in the absence of normal distribution. Female gender was used as reference.

The independent variables used in the multivariate analyses were: age, gender, body mass index (BMI), weight, systolic blood pressure, diastolic blood pressure, mean cIMT, cholesterol (FP, 2-8 h, AUC and AUIC), TAG (FP, 2-8 h, AUC and AUIC), HDL cholesterol (FP, 2-8 h, AUC and AUIC), LDL cholesterol (FP, 2-8 h, AUC and AUIC), VLDL cholesterol (FP, 2-8 h, AUC and AUIC), FFA (FP, 2-8 h, AUC and AUIC), insulin (FP and $4 \mathrm{~h}$ ), CETP (FP and 4 h), FP PLTP, FP HL, FP LPL, oxLDL Ab, and TAG peak oxLDL $A b$. Biphasic and late groups were pooled to reach statistical power.

The tests were considered to be significant at the probability value $(P)$ of 0.05 or less.

\section{Results}

Three groups were observed after the graphic characterization of the responders as early $(65 \%, \mathrm{~N}=39)$, late $(35 \%, \mathrm{~N}=21)$ and biphasic $(\mathrm{N}=10)$ responders. Half of the late responders were biphasic responders.
Early responders were defined as: time to TAG peaks at the 2nd to 4th hour, initial response at the 2nd and 4th hours, back to baseline at the 6th to 8th hour; late responders as: time to TAG peaks at the 6th to 8th hour, initial response at the 2nd to 6th hour and back to baseline, later than in the 8th hour, and biphasic responders as: 1st peak at the 2nd hour and the 2nd peak at the 6th hour.

In the early group, TAG was higher at the 4th hour and lower at the 6th and 8th hours when compared to late responders. FP TAG and the 2 nd-hour responses, as well as peak TAG concentrations, were similar among all groups (Figure 1A).

The early responders showed increased TAG from baseline to the $2 \mathrm{nd}$, 4 th, and 6 th hours; the late and biphasic responders showed increased TAG from baseline to the 2nd, 4th, 6th, and 8th hours (Figure 1A). There were no differences in the magnitude of postalimentary lipemia as measured by the TAG AUCs and AUICs (Figure 1B).

Table 1 shows the similarity of the anthropometric and biochemical characteristics of the participants. No differences were found among the three phenotypes for age, gender, BMI, waist circumference, blood pressure, mean cIMT, TAG, cholesterol, LDL cholesterol, CETP activity, and oxLDL $A b$ in fasting and TAG peak and only in fasting FFA, HL activity, glucose, and insulin. However, the

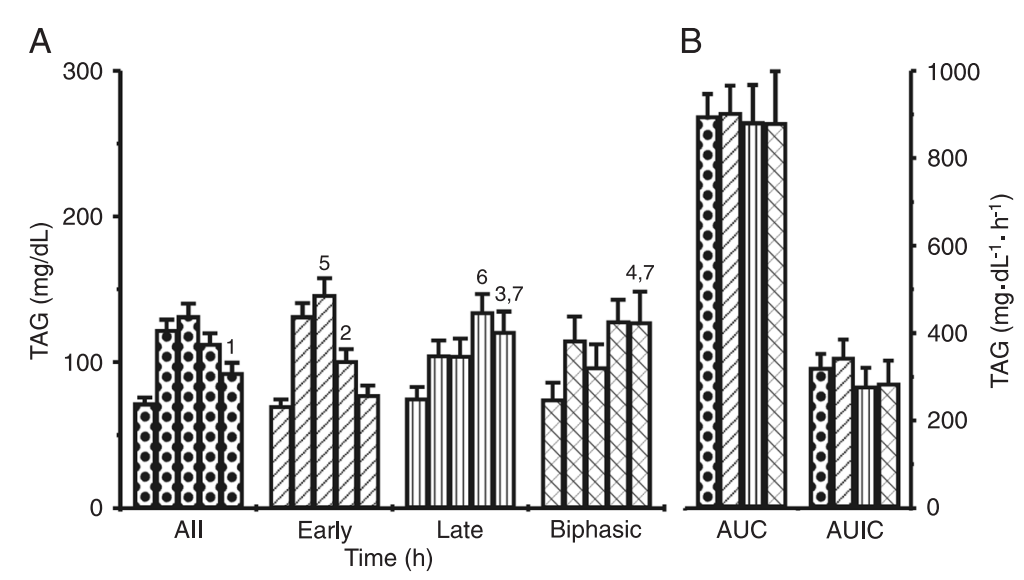

Figure 1. Plasma triacylglycerol (TAG) response groups and triacylglycerol areas under the curves (AUC) and areas under the incremental curves (AUIC) after a standardized fat meal. Data are reported as means \pm SEM. Number of subjects per group: early responders $=39$; late responders $=21$; biphasic responders $=10$. Wilcoxon test. $A$, TAG response groups: during the fasting period (FP, $0 \mathrm{~h}), 2,4$, 6 , and $8 \mathrm{~h}$ by group; all individuals $(\mathrm{N}=60)$ : ${ }^{1} \mathrm{P} \leq 0.0002$, TAG increased from FP to $2,4,6$, and $8 \mathrm{~h}$; early responders: ${ }^{2} \mathrm{P} \leq 0.00001$, TAG increased from fasting to 2, to 4 and to $6 \mathrm{~h}$; late responders: ${ }^{3 P} \leq 0.0033$, TAG increased from FP to 2, 4, 6, and $8 \mathrm{~h}$; biphasic responders: ${ }^{4} \mathrm{P} \leq 0.017$, TAG increased from FP to $2,4,6$, and $8 \mathrm{~h}$. TAG during fasting, at $2 \mathrm{~h}$ and at peaks among groups were not significant; ${ }^{5} \mathrm{P} \leq 0.0169$, TAG at $4 \mathrm{~h}$, higher in early than in late and biphasic responders; ${ }^{6} \mathrm{P}$ $\leq 0.045$, TAG at $6 \mathrm{~h}$, lower in early than in late responders; ${ }^{7} \mathrm{P} \leq 0.0022$, TAG at $8 \mathrm{~h}$, lower in early than in late and biphasic responders (ANOVA followed by the post hoc Duncan test). $B$, TAG AUC and AUIC by group (mg $\left.\cdot \mathrm{dL}^{-1} \cdot \mathrm{h}^{-1}\right)$. Results not significant among groups. 
Table 1. Anthropometric and biochemical characteristics of the participants during the fasting and postalimentary periods.

\begin{tabular}{|c|c|c|c|c|}
\hline \multirow[t]{2}{*}{ Parameters } & \multicolumn{4}{|c|}{ Groups } \\
\hline & All $(\mathrm{N}=60)$ & Early $(\mathrm{N}=39)$ & Late $(\mathrm{N}=21)$ & Biphasic $(N=10)$ \\
\hline Age (years) & $29 \pm 8$ & $30 \pm 7$ & $28 \pm 8$ & $27 \pm 8$ \\
\hline Gender (female/male) & $29 / 31$ & $21 / 18$ & $8 / 13$ & $3 / 7$ \\
\hline Body mass index $\left(\mathrm{kg} / \mathrm{m}^{2}\right)$ & $22 \pm 2$ & $23 \pm 2$ & $22 \pm 2$ & $23 \pm 2$ \\
\hline Waist circumference $(\mathrm{cm})$ & $74 \pm 6$ & $75 \pm 6$ & $73 \pm 7$ & $74 \pm 6$ \\
\hline Systolic blood pressure $(\mathrm{mmHg})$ & $115 \pm 10$ & $113 \pm 10$ & $117 \pm 9$ & $120 \pm 11$ \\
\hline Diastolic blood pressure $(\mathrm{mmHg})$ & $75 \pm 8$ & $74 \pm 8$ & $76 \pm 8$ & $78 \pm 8$ \\
\hline Mean cIMT (mm) & $0.58 \pm 0.06$ & $0.57 \pm 0.06$ & $0.57 \pm 0.06$ & $0.55 \pm 0.04$ \\
\hline FP TAG (mg/dL) & $72 \pm 31$ & $70 \pm 29$ & $75 \pm 35$ & $75 \pm 36$ \\
\hline TAG peak (mg/dL) & $150 \pm 64$ & $153 \pm 66$ & $146 \pm 61$ & $150 \pm 61$ \\
\hline FP cholesterol (mg/dL) & $156 \pm 29$ & $160 \pm 29$ & $150 \pm 29$ & $152 \pm 20$ \\
\hline TAG peak cholesterol (mg/dL) & $155 \pm 30$ & $158 \pm 29$ & $149 \pm 30$ & $150 \pm 20$ \\
\hline FP LDL-C (mg/dL) & $95 \pm 23$ & $96 \pm 22$ & $91 \pm 24$ & $94 \pm 20$ \\
\hline TAG peak LDL-C (mg/dL) & $84 \pm 21$ & $86 \pm 20$ & $81 \pm 22$ & $82 \pm 16$ \\
\hline FP HDL-C (mg/dL) & $49 \pm 11$ & $52 \pm 11$ & $44 \pm 9^{*}$ & $43 \pm 8^{*}$ \\
\hline TAG peak HDL-C (mg/dL) & $47 \pm 12$ & $50 \pm 12$ & $43 \pm 9^{*}$ & $43 \pm 9^{*}$ \\
\hline FP FFA (mM) & $0.7 \pm 0.3$ & $0.7 \pm 0.3$ & $0.7 \pm 0.4$ & $0.7 \pm 0.5$ \\
\hline TAG peak FFA (mM) & $0.7 \pm 0.3$ & $0.6 \pm 0.2$ & $0.9 \pm 0.4^{*}$ & $0.9 \pm 0.5^{\star}$ \\
\hline FP LPL $\left(n m o l ~ F F A \cdot m L-1 \cdot h^{-1}\right)$ & $2303 \pm 792$ & $2114 \pm 789$ & $2667 \pm 682^{*}$ & $3010 \pm 565^{*}$ \\
\hline FP HL (nmol FFA $\left.\cdot \mathrm{mL}^{-1} \cdot \mathrm{h}^{-1}\right)$ & $2861 \pm 1284$ & $2889 \pm 1211$ & $2808 \pm 1457$ & $3249 \pm 1445$ \\
\hline FP CETP $(\%)$ & $17 \pm 6$ & $17 \pm 7$ & $19 \pm 6$ & $19 \pm 4$ \\
\hline TAG peak CETP $(\%)$ & $22 \pm 8$ & $22 \pm 8$ & $22 \pm 8$ & $22 \pm 9$ \\
\hline FP glucose (mg/dL) & $84 \pm 8$ & $85 \pm 8$ & $83 \pm 7$ & $84 \pm 8$ \\
\hline FP insulin $(\mu \mathrm{lU} / \mathrm{mL})$ & $3.5 \pm 1.9$ & $3.8 \pm 2.1$ & $2.8 \pm 1.4$ & $2.4 \pm 0.4$ \\
\hline TAG peak insulin $(\mu \mathrm{lU} / \mathrm{mL})$ & $29 \pm 26$ & $39 \pm 27$ & $10 \pm 9^{*}$ & $8 \pm 6^{*}$ \\
\hline FP oxLDL Ab (\%) & $42 \pm 24$ & $41 \pm 25$ & $44 \pm 24$ & $46 \pm 26$ \\
\hline TAG peak oxLDL Ab (\%) & $51 \pm 25$ & $52 \pm 25$ & $48 \pm 25$ & $39 \pm 15$ \\
\hline
\end{tabular}

Data are reported as means $\pm \mathrm{SD} . \mathrm{N}=$ number of subjects with parameters determined. Early group $=$ time to TAG peaks at the 2 nd to 4 th hour, initial response at the 2 nd and 4 th hours, back to baseline at the 6 th to 8th hour. Late group = time to TAG peaks at the 6th to 8th hour, initial response at the 2nd to 6th hour and back to baseline later than in the 8th hour. Biphasic group $=1$ st peak at the 2nd hour and the 2nd peak at the 6th hour. cIMT = carotid intima-media thickness; TAG = triacylglycerol; FP = fasting period; TAG peak = time of the highest triacylglycerol concentration; LDL-C and HDL-C = low- and high-density lipoprotein cholesterol, respectively; FFA = free fatty acids; $\mathrm{LPL}=$ lipoprotein lipase; $\mathrm{HL}=$ hepatic lipase; $\mathrm{CETP}=$ cholesteryl ester transfer protein; oxLDL $A b=$ autoantibodies to epitopes of oxidized LDL. ${ }^{*} P \leq 0.05$ compared to the early group (chi-square test and ANOVA with post hoc Duncan test).

groups were quite diverse metabolically in the fasting and postalimentary periods.

FP and TAG peak HDL cholesterol were 15 and $17 \%$ lower in late and biphasic responders, respectively, than in early responders; FFA in TAG peak was 33\% lower in the early group than in the late and biphasic responders. FFA was higher in the late and biphasic responders. FP LPL was 21 and $30 \%$ lower in early than in late and biphasic responders, respectively. Insulin in the TAG peak period was 74 and $79 \%$ lower in the late and biphasic groups as compared to the early groups (Table 1 ).
Figure 2A presents postalimentary data about FFA, insulin and CETP for all participants and in the three groups. In early responders, FFA increased from baseline to the 8th hour after the meal and in late responders it decreased from baseline to the 2 nd and the 4 th hours and then increased up to the 8th hour.

All phenotypes increased their insulin concentrations up to their TAG peaks after the meal (Figure 2B). The expected insulin peak would have been in the first $2 \mathrm{~h}$ of the postalimentary period, but the measurements were not determined at this time point. 
CETP in the FP and in the TAG peak period did not differ among groups (Table 1). However, in the early and late responders, CETP activity increased significantly from baseline to TAG peak period after the meal, but not in the biphasic groups (Figure 2C).

Univariate linear regression analysis was performed to test the associations of metabolic and clinical parameters with mean cIMT, FP oxLDL Ab and TAG peak oxLDL Ab in the early and late groups (Table 2 ).

In the early group, we observed significant associations of HL, AUIC TAG, AUIC VLDL cholesterol, and FP insulin (negative) and FP LPL (positive) with TAG peak oxLDLAb, suggesting the main regulators of this biomarker. No associations were observed with FP oxLDL Ab or mean cIMT.

In the late group, the mean cIMT was positively associated with cholesterol (FP, time 2-8 $\mathrm{h}$ and AUC), TAG (FP, time 2-8 $\mathrm{h}$ and AUC), LDL cholesterol (FP, time 2-8 $\mathrm{h}$ and $A U C$ ) and VLDL cholesterol (FP, time 2-8 $\mathrm{h}$ and $A U C$ ). FP oxLDL Ab was positively associated with FFA $2 \mathrm{~h}$ and negatively associated with male gender, while the TAG peak oxLDLAb in the late group was positively associated with AUIC cholesterol and AUIC FFA (Table 2). These associations suggest that lipids and autoantibodies against epitopes of oxidized LDL may exert an important effect on early atherosclerosis.

Multivariate analysis was then performed to confirm the associations with mean cIMT and oxLDLAb (Table 3). In the early group, mean cIMT was positively explained by male gender and age, as described earlier, while in the late group cholesterol at the 6th hour was the cIMT regulator. In the late group the FP oxLDL Ab showed positive associations with FFA $2 \mathrm{~h}$ but negative associations with CETP at the 4th hour, indicating the anti-oxidant role of CETP.

Regarding the TAG peak oxLDL $A b$, the early group showed positive associations with FP LPL and inverse associations with $\mathrm{HL}$, while in the late group only AUIC cholesterol was positively associated. These lipases have ambiguous roles in atherogenesis $(22,23)$.

Analysis of ApoE polymorphisms $(\mathrm{N}=44)$ as determinants of postalimentary lipemia showed that 14,57 , and $29 \%$ of the subjects presented $\varepsilon 2, \varepsilon 3$, and $\varepsilon 4$ alleles, respectively. The distribution among apolipoprotein $E$ genotypes was similar among all responders $(P=0.667)$.

\section{Discussion}

The variations of lipids and lipoproteins in response to a fat meal were measured in order to detect associations with clinical and circulating atherosclerosis biomarkers.

A phenomenon of fat carry-over from a previous meal to the postalimentary period of the subsequent meal has been described by several investigators (24) to explain the
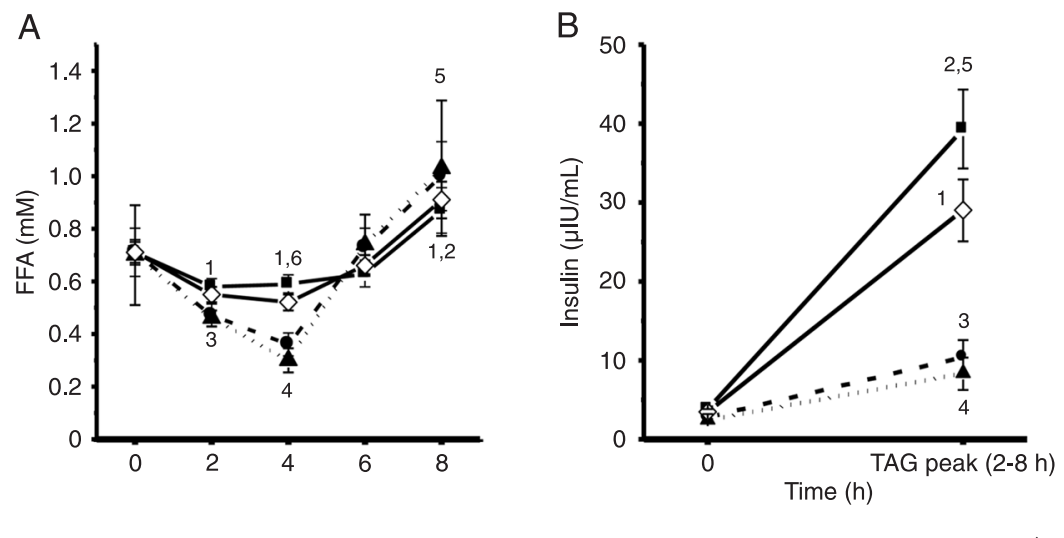

Early

- Late

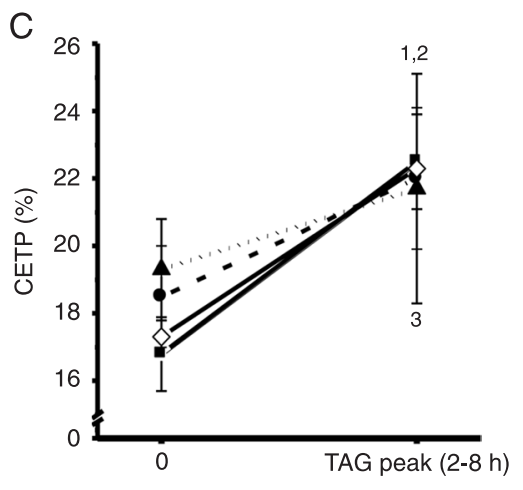

$\Delta$ Biphasic $\diamond$ All

Figure 2. Postalimentary plasma free fatty acids (FFA), insulin and cholesteryl ester transfer protein (CETP) after a standardized meal. Data are reported as means $\pm \mathrm{SEM}$. $\mathrm{N}=$ minimum to maximum number of subjects with parameters determined. Wilcoxon and ANOVA with post hoc Duncan tests. A, FFA: all individuals [N = 54 subjects in the fasting period (FP), 4th and 8th hours; $N=52$ at the $2 \mathrm{nd}$ hour and $\mathrm{N}=51$ at the 6 th hour], ${ }^{1} \mathrm{P} \leq 0.008$, FFA decreased from baseline to the 2 nd and 4 th hours, and increased to the 8 th hour; early responders ( $N=37$ subjects in the FP and at the 4th and 8th hours; $N=35$ at 2 nd hour and $N=34$ at 6 th hour), ${ }^{2} \mathrm{P} \leq 0.045$, FFA increased from baseline to the 8th hour; late responders ( $N=17$, all times), ${ }^{3} \mathrm{P} \leq 0.04$, FFA decreased from baseline to the $2 \mathrm{nd}$ hour, ${ }^{4} \mathrm{P} \leq 0.005$ to the 4 th hour, and ${ }^{5} \mathrm{P} \leq 0.012 \mathrm{FFA}$ increased to the 8 th hour; early responders: ${ }^{6} \mathrm{P} \leq 0.00001$ at the 4 th hour higher than late and biphasic responders $\left(N=7\right.$, all times). $B$, Insulin: ${ }^{1} P \leq 0.00001,{ }^{2} P \leq 0.00001,{ }^{3} P \leq 0.0009,{ }^{4} P \leq 0.018$, respectively, in all individuals ( $N=47$ in FP and $N=45$ in TAG peak), early responders $(N=31$ in FP and $N=29$ in TAG peak), late responders ( $N=$ 16 in FP and TAG peak), and biphasic responders ( $N=7$ in FP and TAG peak), insulin increased from baseline to the 4th hour; early responders: ${ }^{5 P} \leq 0.00001$, higher peak than in late and biphasic responders. $C$, CETP: in all individuals $(N=54$ subjects in FP and $\mathrm{N}=48$ in TAG peak), early responders ( $N=37$ in FP and $N=33$ in TAG peak) and late responders ( $N=17$ in FP and $N=15$ in TAG peak), CETP increased from baseline to the 4 th hour: ${ }^{1} \mathrm{P} \leq 0.0003,{ }^{2} \mathrm{P} \leq 0.002,{ }^{3} \mathrm{P} \leq 0.044$, respectively (Wilcoxon test). Biphasic ( $\mathrm{N}=$ 7 subjects in FP and TAG peak): results not significant at 4th hour. 
Table 2. Univariate linear regression analysis for subclinical carotid atherosclerosis and antibodies against oxidized LDL in early and late responders.

\begin{tabular}{|c|c|c|c|}
\hline & Beta (SE) & $\mathrm{P}$ & $\mathrm{R}^{2}$ \\
\hline \multicolumn{4}{|l|}{ Early group ( $N=26$ ) } \\
\hline \multicolumn{4}{|l|}{ TAG peak oxLDL Ab } \\
\hline FP HL & $-0.43(0.17)$ & 0.021 & 0.2015 \\
\hline FP LPL & $0.47(0.16)$ & 0.006 & 0.2767 \\
\hline AUIC TAG & $-0.37(0.16)$ & 0.028 & 0.1864 \\
\hline AUIC VLDL-C & $-0.37(0.16)$ & 0.028 & 0.1861 \\
\hline FP insulin & $-0.40(0.17)$ & 0.025 & 0.1914 \\
\hline \multicolumn{4}{|l|}{ Late group§ $(N=14)$} \\
\hline \multicolumn{4}{|l|}{ Mean clMT } \\
\hline Gender (male) & $2.94(2.07)$ & 0.184 & 0.1548 \\
\hline Age (years) & $0.34(0.23)$ & 0.161 & 0.1701 \\
\hline FP cholesterol & $0.57(0.17)$ & 0.008 & 0.4908 \\
\hline Cholesterol $2 \mathrm{~h}$ & $0.59(0.19)$ & 0.011 & 0.4621 \\
\hline Cholesterol $4 \mathrm{~h}$ & $0.59(0.18)$ & 0.007 & 0.5035 \\
\hline Cholesterol $6 \mathrm{~h}$ & $0.69(0.15)$ & $<0.001$ & 0.6676 \\
\hline Cholesterol $8 \mathrm{~h}$ & $0.63(0.18)$ & 0.005 & 0.5240 \\
\hline AUC cholesterol & $0.60(0.18)$ & 0.006 & 0.5096 \\
\hline FP TAG & $0.58(0.17)$ & 0.006 & 0.5057 \\
\hline TAG $2 \mathrm{~h}$ & $0.59(0.18)$ & 0.008 & 0.4828 \\
\hline TAG $4 \mathrm{~h}$ & $0.51(0.20)$ & 0.028 & 0.3696 \\
\hline TAG $6 \mathrm{~h}$ & $0.57(0.17)$ & 0.006 & 0.5085 \\
\hline TAG $8 \mathrm{~h}$ & $0.58(0.19)$ & 0.012 & 0.4546 \\
\hline AUC TAG & $0.60(0.17)$ & 0.005 & 0.5277 \\
\hline FP LDL-C & $0.62(0.16)$ & 0.003 & 0.5708 \\
\hline LDL-C 2 h & $0.61(0.17)$ & 0.004 & 0.5485 \\
\hline LDL-C $4 \mathrm{~h}$ & $0.56(0.18)$ & 0.009 & 0.4821 \\
\hline LDL-C $6 \mathrm{~h}$ & $0.58(0.17)$ & 0.007 & 0.5013 \\
\hline LDL-C 8 h & $0.57(0.18)$ & 0.008 & 0.4839 \\
\hline AUC LDL-C & $0.57(0.18)$ & 0.008 & 0.4889 \\
\hline FP VLDL-C & $0.59(0.18)$ & 0.007 & 0.5026 \\
\hline VLDL-C 2 h & $0.59(0.18)$ & 0.007 & 0.4952 \\
\hline VLDL-C $4 \mathrm{~h}$ & $0.53(0.20)$ & 0.020 & 0.4023 \\
\hline VLDL-C $6 \mathrm{~h}$ & $0.57(0.17)$ & 0.006 & 0.5085 \\
\hline VLDL-C 8 h & $0.61(0.19)$ & 0.008 & 0.4919 \\
\hline AUC VLDL-C & $0.61(0.17)$ & 0.004 & 0.5453 \\
\hline \multicolumn{4}{|l|}{ FP oxLDL Ab } \\
\hline Gender (male) & $-4.67(1.93)$ & 0.032 & 0.3282 \\
\hline FFA $2 \mathrm{~h}$ & $0.71(0.16)$ & $<0.001$ & 0.6140 \\
\hline \multicolumn{4}{|l|}{ TAG peak oxLDL Ab } \\
\hline AUIC cholesterol & $0.58(0.21)$ & 0.016 & 0.3939 \\
\hline AUIC FFA & $0.58(0.21)$ & 0.018 & 0.3864 \\
\hline
\end{tabular}

Beta $(\mathrm{SE})=$ value of the slope in the regression line (standard error); $\mathrm{R}^{2}=$ coefficient of determination. For explanation of early and late groups, see legend to Table 1. FP = fasting period; oxLDL Ab = autoantibodies to epitopes of oxidized LDL; TAG = triacylglycerol; cIMT = carotid intima-media thickness; $\mathrm{HL}=$ hepatic lipase; $\mathrm{LPL}=$ lipoprotein lipase; $A U I C=$ areas under the incremental curves; VLDL-C and LDL-C = very low-density and low-density lipoprotein cholesterol, respectively; AUC = areas under the curve; FFA = free fatty acids. $\S$ Late and biphasic responders were pooled. behavior of late and biphasic responders (Figure 1; Table 1) These chylomicrons might be either stored in enterocytes or in the lymphatic vessels (25). Since in the present study the biphasic participants had fasted for $12 \mathrm{~h}$ before the liquid meal, it is not likely that they had stored chylomicrons from a previous meal, but rather that chylomicrons were formed following the more recent ingestion. The second peak at the 6th hour could be explained by increased VLDL production and competition for clearance with chylomicrons in the postalimentary period (26). The extension of TAG-rich lipoprotein residence times in contrast to early responders can generate substrate availability for oxidation of VLDL and LDL, thereby inducing a local inflammatory stimulus, generation of autoantibodies to LDL epitopes and plaque formation in the artery wall (27).

Late and biphasic responders presented lower fasting and TAG peak plasma HDL cholesterol (16\%) compared to early responders (Table 1). Since HDL has several effects on the inhibition of atherogenesis, such as a role in reverse cholesterol transport, acting as an antioxidant, a profibrinolytic or an antithrombogenic agent, its reduction is implicated in an increase in the risk of atherosclerosis (28).

On the other hand, there is also an inverse relationship between circulating HDL cholesterol and TAG, but in this study fasting triacylglycerol levels did not differ between the three groups. This was surprising because fasting hypertriglyceridemia has been identified as the strongest determinant of postprandial lipemia (29). It has been reported that subjects with reduced fasting plasma HDL cholesterol levels are characterized by increased postalimentary lipemia. Therefore, the described observation of the postalimentary state phenotypes could possibly be an HDL effect as well as an effect of response time.

Cholesteryl ester transfer protein is a plasma lipid transfer protein that has the ability to transfer cholesteryl esters from HDL to apolipoprotein B-containing lipoproteins playing an important role in reverse cholesterol transport. The absent CETP response in biphasic individuals during the postalimentary period would favor the accumulation of TAG-rich lipoproteins. When CETP activity is reduced, the transfer of lecithin:cholesterol acyltransferase-derived cholesteryl esters from HDL to other lipoprotein fractions is reduced and as a result the total core lipid content and the particle surface of HDL increase (30). In addition, the clearance of TAG-rich lipoproteins is reduced. This agrees with the higher TAG at the 6th and 8th hours after the meal in the late group. The mechanism(s) underlying the reduction of CETP activity in late responders is unknown but could be secondary to chemical modifications of postalimentary HDL particles.

During the TAG peak period the insulin concentration was much lower $(-77 \%)$ in late responders than in the early group (Table 1; Figure 2). We propose that the diminished response is a metabolic consequence of previous meals due to a chronic and regular proximity between them and their 
synergistic effects. The response profile is more similar to the one of the fasting period. In accordance with this hypothesis no TAG peaks were observed at the 4 th hour and fasting LPL activity was higher (26\%) in late responders, reflecting a previous meal effect. Furthermore, the similar TAG in the early and late groups reflects increased lipolysis in the latter group.

In early responders, FFA concentrations increased up to the 8th hour after the meal (Table 1; Figure 2). In late and biphasic responders, despite the reduced insulin concentrations, a paradoxical $43 \%$ decrease in FFA concentration was observed at the 4th hour and an increase again up to the 8th hour. The decrease in FFA is suggestive of a stimulated tissue uptake or a hyperresponsiveness to insulin. In addition, a decreased postalimentary lipolysis mediated by lipoprotein lipase could be ruled out, although the lipase activities were not measured in the postalimentary period. Moreover, the decreased insulin concentration observed in these late responders in the postalimentary period could favor TAG lipolysis in the adipose tissue and a consequent increase in plasma FFA concentration; consequently, an increased liver production of VLDL with a decreased lipolysis rate of TAG-rich lipoproteins would occur. This agrees with the observations of a higher 6th- and 8th-hour TAG in the late group.

Subclinical carotid atherosclerosis has been related to age, gender, smoking, high cholesterol and LDL cholesterol, triglycerides, and postalimentary lipemia (31).

In the late group, the univariate positive associations with mean cIMT with age, gender and all lipid profile components and their respective AUC at all times, with the exception of HDL cholesterol, suggest the presence of an atherogenic lipid profile in the postalimentary period. Corroborating this fact, we observed positive associations in the FP of oxLDL $\mathrm{Ab}$ with FFA at $2 \mathrm{~h}$ and male gender, and in the TAG peak with cholesterol and FFAAUIC.

In the early group, TAG peak oxLDL Ab was inversely related to TAG and VLDL cholesterol AUIC and insulin, suggesting physiologic response patterns. Its negative association with $\mathrm{HL}$ and positive association with LPL indicate opposite roles of these lipases in the generation of postalimentary oxidized lipids, discussed with the subsequent multivariate analysis.

In the definitive multivariate analysis, as expected, the early responders' mean cIMT was explained by gender (males) and age and in late responders by cholesterol levels at the 6th hour, $146 \pm 32 \mathrm{mg} / \mathrm{dL}$ (data not shown), values very similar to the TAG peak cholesterol $(149 \pm 30$ $\mathrm{mg} / \mathrm{dL})$. sponders.
Table 3. Relationships of early carotid atherosclerosis and antibodies against

Beta (SE) P Partial $R^{2}$

\begin{tabular}{lll}
$8.35(3.00)$ & 0.011 & 0.1661 \\
$0.37(0.17)$ & 0.044 & 0.1375 \\
& & \\
$0.42(0.15)$ & 0.008 & 0.2767 \\
$-0.36(0.15)$ & 0.029 & 0.1378 \\
& & \\
$0.75(0.15)$ & $<0.001$ & 0.7113 \\
& & \\
$0.68(0.14)$ & $<0.001$ & 0.6140 \\
$-0.35(0.16)$ & 0.048 & 0.1199 \\
& & \\
$0.58(0.21)$ & 0.016 & 0.3939 \\
\hline
\end{tabular}

Beta $(\mathrm{SE})=$ value of the slope in the regression line (standard error); $\mathrm{R}^{2}=$ coefficient = hepatic lipase AUIC = areas under the incremental curves. §Late and biphasic

Interestingly, in early responders $\mathrm{LPL}$ and $\mathrm{HL}$ regulated the titers of antibodies against oxidized LDL in a positive and negative manner, respectively.

The increased LPL activity implies a higher generation of FFA, and it is well known that different fatty acids liberated during lipolysis may have different actions on inflammation. Omega- 6 polyunsaturated fatty acids promote inflammation, whereas the omega-3 family has anti-inflammatory properties such as inhibiting cytokine production in leukocytes (32), decreasing NF-KB activation and IL-1, IL-6, and TNF- $\alpha$ mRNA expression in different experimental models (33). The positive regulation of oxLDL $A b$ by LPL agrees with $a$ publication that attributed an inflammatory function to LPL (22). Another interesting fact is that the macrophage LPL locally releases fatty acids that may serve as an energy source, but is also a potential pro-inflammatory stimulus for these cells by the formation of lipid droplets, inducing endoplasmic reticulum stress $(34,35)$.

Hepatic lipase has dual functions as a triglyceride hydrolase and ligand/bridging factor for receptor-mediated lipoprotein uptake. Consistently, human and animal studies support antiatherogenic (36) and pro-atherogenic roles for hepatic lipase since genetically low or absent HL activity can be associated with increased CAD risk (23). The genetic variation of the $\mathrm{HL}$ gene is associated with TAG and lipoproteins containing elevated apoB levels in humans (37). The negative association with $\mathrm{HL}$ agrees with the increase in these lipoprotein levels and possibly with the higher generation of oxidized LDL and its autoantibodies. 
In late responders, oxLDL Ab was positively regulated by FFA and negatively regulated by CETP. As discussed before, the FFA released in lipolysis can act as an inflammatory stimulus, indirectly explaining the positive association with the antibodies against oxLDL.

In animal models of acute inflammation, CETP was shown to act as a first line of defense by reducing the exacerbated production of TNF- $\alpha$ and IL-6, both pro-inflammatory mediators (38). CETP can also decrease the macrophage cholesteryl ester content and decrease the circulating oxidized LDL (39); so, the negative association of CETP activity with oxLDL Ab during the fasting period reinforces a pro-atherogenic character of CETP reduction, even after the changes caused by the postprandial state.

This study is the first to provide a characterization of a possible postalimentary insulin resistance state combined with a reduced CETP response - and possible decreased reverse cholesterol transport - exclusive to late responders, and the identification of the regulators of postalimentary atherogenicity, although the number of individuals studied was limited and no mechanisms were studied in depth.

The data presented in this study suggest that in asymptomatic adults a fat diet can produce higher or lower potential

\section{References}

1. McBride PE. Triglycerides and risk for coronary heart disease. JAMA 2007; 298: 336-338.

2. Hokanson JE, Austin MA. Plasma triglyceride level is a risk factor for cardiovascular disease independent of highdensity lipoprotein cholesterol level: a meta-analysis of population-based prospective studies. J Cardiovasc Risk 1996; 3: 213-219.

3. Roche HM, Gibney MJ. The impact of postprandial lipemia in accelerating atherothrombosis. J Cardiovasc Risk 2000; 7: 317-324.

4. Bansal S, Buring JE, Rifai N, Mora S, Sacks FM, Ridker PM. Fasting compared with nonfasting triglycerides and risk of cardiovascular events in women. JAMA 2007; 298: 309316.

5. Nordestgaard BG, Benn M, Schnohr P, Tybjaerg-Hansen A. Nonfasting triglycerides and risk of myocardial infarction, ischemic heart disease, and death in men and women. JAMA 2007; 298: 299-308.

6. Proctor SD, Vine DF, Mamo JC. Arterial retention of apolipoprotein $\mathrm{B}(48)$ - and $\mathrm{B}(100)$-containing lipoproteins in atherogenesis. Curr Opin Lipidol 2002; 13: 461-470.

7. Patel S, Puranik R, Nakhla S, Lundman P, Stocker R, Wang $X S$, et al. Acute hypertriglyceridaemia in humans increases the triglyceride content and decreases the anti-inflammatory capacity of high density lipoproteins. Atherosclerosis 2009; 204: 424-428.

8. Zilversmit DB. Atherogenesis: a postprandial phenomenon. Circulation 1979; 60: 473-485.

9. Sniderman AD. Postprandial hypertriglyceridemia(s): time to enlarge our pathophysiologic perspective. Eur J Clin Invest 2000; 30: 935-937. atherogenic changes depending on the individual response phenotype. These results call attention to the fact that in primary atherosclerosis prevention individuals presenting predisposing and risk factors for atherosclerosis (such as metabolic syndrome, obesity, sedentariness, smoking, and hypertension) an increased postprandial response would occur, leading to further atherosclerotic repercussions.

Further research is required to determine the metabolic mechanisms described in the different postalimentary phenotypes observed in the present study, as well as in different pathological states, as currently investigated in our laboratory.

\section{Acknowledgments}

The authors acknowledge the excellent technical and clinical assistance provided by Dr. Denise Kaplan, Miriam Danelon and Aparecida de Sousa, as well as the support by Dr. Edna Nakandakare and the Laboratory of Lipids, Medical School of the University of São Paulo. Research supported in part by FAPESP (\#03212-5), CNPq (\#473185), and CAPES.

10. Patsch JR, Miesenbock G, Hopferwieser T, Muhlberger V, Knapp E, Dunn JK, et al. Relation of triglyceride metabolism and coronary artery disease. Studies in the postprandial state. Arterioscler Thromb 1992; 12: 1336-1345.

11. Boquist S, Ruotolo G, Tang R, Bjorkegren J, Bond MG, de Faire $U$, et al. Alimentary lipemia, postprandial triglyceriderich lipoproteins, and common carotid intima-media thickness in healthy, middle-aged men. Circulation 1999; 100: 723-728.

12. Karpe F, de Faire $U$, Mercuri M, Bond MG, Hellenius ML, Hamsten A. Magnitude of alimentary lipemia is related to intima-media thickness of the common carotid artery in middle-aged men. Atherosclerosis 1998; 141: 307-314.

13. Lorenz MW, Markus HS, Bots ML, Rosvall M, Sitzer M. Prediction of clinical cardiovascular events with carotid intimamedia thickness: a systematic review and meta-analysis. Circulation 2007; 115: 459-467.

14. Tsai WC, Li YH, Chao TH, Chen JH. Relation between antibody against oxidized low-density lipoprotein and extent of coronary atherosclerosis. J Formos Med Assoc 2002; 101: 681-684

15. Executive Summary of the Third Report of the National Cholesterol Education Program (NCEP) Expert Panel on Detection, Evaluation, and Treatment of High Blood Cholesterol in Adults (Adult Treatment Panel III). JAMA 2001; 285: 2486-2497.

16. Ehnholm C, Kuusi T. Preparation, characterization, and measurement of hepatic lipase. Methods Enzymol 1986; 129: 716-738.

17. Lagrost L. Determination of the mass concentration and the activity of the plasma cholesteryl ester transfer protein 
(CETP). Methods Mol Biol 1998; 110: 231-241.

18. Gidlund M, Damasceno NR, Lindoso JA, Abdalla DS, Goto $\mathrm{H}$. Monoclonal antibodies against low density lipoprotein with various degrees of oxidative modifications. Braz J Med Biol Res 1996; 29: 1625-1628.

19. Salazar LA, Hirata MH, Cavalli SA, Machado MO, Hirata RD. Optimized procedure for DNA isolation from fresh and cryopreserved clotted human blood useful in clinical molecular testing. Clin Chem 1998; 44: 1748-1750.

20. Emi M, Wu LL, Robertson MA, Myers RL, Hegele RA, Williams RR, et al. Genotyping and sequence analysis of apolipoprotein E isoforms. Genomics 1988; 3: 373-379.

21. Carallo C, Irace C, Pujia A, De Franceschi MS, Crescenzo A, Motti C, et al. Evaluation of common carotid hemodynamic forces. Relations with wall thickening. Hypertension 1999; 34: 217-221.

22. Higgins LJ, Rutledge JC. Inflammation associated with the postprandial lipolysis of triglyceride-rich lipoproteins by lipoprotein lipase. Curr Atheroscler Rep 2009; 11: 199-205.

23. Jansen H, Verhoeven AJ, Sijbrands EJ. Hepatic lipase: a pro- or anti-atherogenic protein? J Lipid Res 2002; 43: 1352-1362.

24. Fielding BA, Callow J, Owen RM, Samra JS, Matthews DR, Frayn KN. Postprandial lipemia: the origin of an early peak studied by specific dietary fatty acid intake during sequential meals. Am J Clin Nutr 1996; 63: 36-41.

25. Mattes RD. Oral fat exposure increases the first phase triacylglycerol concentration due to release of stored lipid in humans. J Nutr 2002; 132: 3656-3662.

26. Robins SJ, Fasulo JM, Robins VF, Patton GM. Response of serum triglycerides of endogenous origin to the administration of triglyceride-rich lipid particles. Am J Physiol 1989; 257: E860-E865.

27. Le NA, Li X, Kyung S, Brown WV. Evidence for the in vivo generation of oxidatively modified epitopes in patients with atherosclerotic endothelium. Metabolism 2000; 49: 12711277.

28. Spieker LE, Ruschitzka F, Luscher TF, Noll G. HDL and inflammation in atherosclerosis. Curr Drug Targets Immune Endocr Metabol Disord 2004; 4: 51-57.

29. Kolovou GD, Anagnostopoulou KK, Pavlidis AN, Salpea KD, Iraklianou SA, Hoursalas IS, et al. Metabolic syndrome and gender differences in postprandial lipaemia. Eur J Cardiovasc Prev Rehabil 2006; 13: 661-664.

30. Parra ES, Urban A, Panzoldo NB, Nakamura RT, Oliveira R, de Faria EC. A reduction of CETP activity, not an increase, is associated with modestly impaired postprandial lipemia and increased HDL-cholesterol in adult asymptomatic women. Lipids Health Dis 2011; 10: 87.

31. Fukumoto $M$, Shoji $T$, Emoto $M$, Kawagishi $T$, Okuno $Y$, Nishizawa Y. Antibodies against oxidized LDL and carotid artery intima-media thickness in a healthy population. Arterioscler Thromb Vasc Biol 2000; 20: 703-707.

32. Pompeia C, Lopes LR, Miyasaka CK, Procopio J, Sannomiya P, Curi R. Effect of fatty acids on leukocyte function. Braz J Med Biol Res 2000; 33: 1255-1268.

33. Fernandes G, Bhattacharya A, Rahman M, Zaman K, Banu J. Effects of n-3 fatty acids on autoimmunity and osteoporosis. Front Biosci 2008; 13: 4015-4020.

34. den Hartigh LJ, Connolly-Rohrbach JE, Fore S, Huser TR, Rutledge JC. Fatty acids from very low-density lipoprotein lipolysis products induce lipid droplet accumulation in human monocytes. J Immunol 2010; 184: 3927-3936.

35. Lichtenstein L, Mattijssen F, de Wit NJ, Georgiadi A, Hooiveld GJ, van der Meer R, et al. Angpt/4 protects against severe proinflammatory effects of saturated fat by inhibiting fatty acid uptake into mesenteric lymph node macrophages. Cell Metab 2010; 12: 580-592.

36. Karackattu SL, Trigatti B, Krieger M. Hepatic lipase deficiency delays atherosclerosis, myocardial infarction, and cardiac dysfunction and extends lifespan in SR-Bl/apolipoprotein E double knockout mice. Arterioscler Thromb Vasc Biol 2006; 26: 548-554.

37. Connelly PW, Hegele RA. Hepatic lipase deficiency. Crit Rev Clin Lab Sci 1998; 35: 547-572.

38. Cazita PM, Barbeiro DF, Moretti AI, Quintao EC, Soriano FG. Human cholesteryl ester transfer protein expression enhances the mouse survival rate in an experimental systemic inflammation model: a novel role for CETP. Shock 2008; 30: 590-595.

39. Oliveira HC, de Faria EC. Cholesteryl ester transfer protein: the controversial relation to atherosclerosis and emerging new biological roles. IUBMB Life 2011; 63: 248-257. 